\title{
Qualidade de vida em voz: o impacto de uma disfonia de acordo com gênero, idade e uso vocal profissional
}

\author{
Voice-related quality of life: the impact of a dysphonia \\ according to gender, age and occupational use of voice
}

\author{
Daniele de Souza Putnoki ${ }^{1}$, Fabiana Hara ${ }^{2}$, Gisele Oliveira ${ }^{3}$, Mara Behlau ${ }^{4}$
}

\begin{abstract}
RESUMO
Objetivo: Verificar o impacto auto-relatado de uma alteração vocal na qualidade de vida de indivíduos com queixa de voz, de acordo com gênero, idade e uso vocal profissional. Métodos: Foram analisados 1304 questionários - Qualidade de Vida em Voz (QVV) - respondidos por brasileiros com queixa vocal variada, sendo 996 mulheres e 308 homens. No tratamento estatístico foram feitas comparações entre gênero, idade e nível de uso vocal profissional. Resultados: Os resultados totais foram similares nos três domínios para homens (total 75,5; físico 71,3; sócio-emocional 82,3) e mulheres (total 74,9; físico 70,7; sócio-emocional 82,1). Observaram-se maiores escores do QVV nos indivíduos entre 20 e 29 anos (total 82,2; físico 77,8; sócio-emocional 89,2). De acordo com o nível de uso da voz, profissionais do nível I apresentaram os maiores índices (total 80,93 e físico 80,97). Conclusão: O impacto auto-relatado na qualidade de vida relacionado a uma alteração vocal foi percebido de maneira semelhante por homens e mulheres. Os indivíduos de 20 a 29 anos perceberam o impacto da disfonia na sua qualidade de vida diferente dos indivíduos das outras faixas etárias. Os profissionais com grande demanda de voz falada artística relataram sofrer o menor impacto de um problema de voz na qualidade de vida.
\end{abstract}

Descritores: Voz; Qualidade de vida; Distúrbios da voz; Disfonia; Perfil de impacto da doença; Cuidados de saúde; Avaliação em saúde; Efeito idade

\section{INTRODUÇÃO}

De acordo com a Organização Mundial de Saúde (WHO, 1997) o conceito de saúde não se limita à ausência de doença. Em sua definição inclui-se o aspecto de qualidade de vida, que é um conceito amplo e complexo e que engloba características subjetivas e multidimensionais, considerando as percepções individuais positivas e negativas do estado físico, psicológico e social, a posição na vida, o contexto cultural e sistema de valores em que o sujeito vive, relacionado a seus objetivos, expectativas, padrões e preocupações ${ }^{(1)}$.

Tradicionalmente na área da saúde, incluindo a Fonoaudiologia, os testes objetivos e resultados clínicos são a forma do

Trabalho realizado no Centro de Estudos da Voz - CEV - São Paulo (SP), Brasil.

(1) Fonoaudióloga clínica - Vinhedo (SP), Brasil.

(2) Fonoaudióloga do Hospital Beneficência Portuguesa - São Paulo (SP), Brasil.

(3) Doutora, Professora do Curso de Especialização em Voz do Centro de Estudos da Voz - CEV - São Paulo (SP), Brasil.

(4) Doutora, Professora do Curso de Especialização em Voz do Centro de Estudos da Voz - CEV - São Paulo (SP), Brasil.

Endereço para corrrespondência: Daniele de Souza Putnoki. Al. Jaburu, 297, Vista Alegre, Vinhedo (SP), Brasil, CEP: 13280-000. E-mail: daniputnoki@gmail.com

Recebido em: 24/11/2008; Aceito em: 17/12/2009 profissional acessar as mudanças nos pacientes. Embora essas informações sejam importantes no processo patológico, elas não mostram o ponto de vista do paciente sobre sua doença e suas perspectivas profissionais e sociais ${ }^{(2-4)}$.

O impacto de uma alteração vocal na qualidade de vida depende da importância da voz relacionada a diversos fatores particulares, inclusive seu uso na profissão, sem necessariamente apresentar relação direta com o grau da disfonia ${ }^{(5)}$. O estresse psicológico é um exemplo de consequência, que implica em piora da qualidade de vida social, da pessoa com problema de $\mathrm{voz}^{(6)}$.

O uso da mensuração de qualidade de vida como método de avaliação dos resultados do tratamento em pacientes com disfonia vem aumentando ${ }^{(7)}$. Isso permite diferenciar pacientes ou agrupá-los, prognosticar resultados individuais, avaliar a efetividade da terapia, além de ajudar o profissional a priorizar problemas, comunicar-se melhor com seu paciente, proteger-se de adversidades e identificar as preferências do paciente ${ }^{(2,4)}$.

Uma forma de avaliar a qualidade de vida na área da saúde é por meio dos questionários de auto-avaliação, com perguntas relacionadas ao comportamento, sentimentos e $\operatorname{sintomas}^{(3,8)}$. Pesquisadores têm se dedicado ao desenvolvimento de instrumentos que visam investigar a percepção do paciente sobre o impacto causado pela disfonia em sua qualidade de $\operatorname{vida}^{(9)}$. 
O Voice-related Quality of Life (V-RQOL) $)^{(8)}$, é um protocolo de auto-avaliação, que investiga o impacto de uma disfonia na vida do indivíduo. É um questionário clínico que vem sendo amplamente utilizado $^{(8,10,11)}$, foi traduzido ${ }^{(12)}$ e validado ${ }^{(11,13-16)}$ para o Português com o nome de Qualidade de Vida em Voz $(\mathrm{QVV})^{(10)}$. Há inclusive uma adaptação deste questionário para a população pediátrica ${ }^{(16,17)}$.

O protocolo QVV permite estudar o impacto de um problema de voz na qualidade de vida de indivíduos disfônicos. A literatura neste assunto é vasta, contudo há poucas informações sobre a influência do gênero, idade e uso vocal profissional na percepção desse impacto.

Há dados que parecem indicar influência do gênero no desenvolvimento de uma disfonia, como o fato de as mulheres apresentarem maior predisposição para desenvolver problemas de voz, a laringe feminina é menor e a proporção glótica é reduzida em relação à masculina, e por representarem maior parte da população que procura por atendimento fonoaudiológico clínico ${ }^{(5,18)}$.

A importância de se pesquisar a percepção de um possível impacto da voz na qualidade de vida, em diferentes faixas etárias, deve-se ao fato da voz não ser uma manifestação constante e sim um produto com características específicas na adolescência, fase adulta e senescência ${ }^{(19,20)}$, além de ser um marcador extremamente sensível à fase de maior produtividade profissional $^{(21-23)}$.

A voz é um instrumento essencial na vida de diversos profissionais ${ }^{(24,25)}$, sendo que seu uso pode ser diferente de acordo com a profissão tanto na quantidade quanto na forma. Por exemplo, professores e operadores de teleatendimento, que fazem uso da voz profissional não artística, têm grande demanda vocal; o mesmo acontece com cantores e atores que também utilizam muito a voz, porém com características artísticas. Outros profissionais têm menor demanda vocal em seu trabalho, como advogados e alguns não precisam utilizar a voz, como programadores de computação ${ }^{(18)}$.

Acredita-se que o uso de protocolos que avaliam qualidade de vida relacionada à voz, como o QVV, em grandes populações com queixa vocal pode fornecer a identificação de diferenças na percepção do impacto de uma disfonia de acordo com o gênero, faixa etária e uso vocal profissional.

Assim, o objetivo deste estudo foi verificar o impacto auto-relatado de uma alteração vocal na qualidade de vida de indivíduos com queixa de voz, de acordo com o gênero, idade e uso vocal profissional.

\section{MÉTODOS}

Este trabalho foi aprovado pelo Comitê de Ética do Centro de Estudos da Voz - CEV (0815/07) e os participantes voluntários consentiram em responder ao protocolo.

Para este estudo foram analisados 2325 protocolos QVV, que foram aplicados por fonoaudiólogos de todo o Brasil, vinculados à instituição em que o estudo foi realizado e que receberam instruções sobre o conceito de qualidade de vida e treino prático para a aplicação do questionário. Houve a exclusão de 111 questionários por falta de dados completos, levando a um total de 2214 participantes. Os 2214 protocolos restantes foram divididos em dois grupos, de acordo com a presença ou não de queixa vocal. Para esta investigação foi considerado apenas o grupo com queixa e diagnóstico de alteração vocal, compondo uma amostra de 1304 indivíduos (996 mulheres e 308 homens) com idades entre 14 e 80 anos e média de 34 anos e cinco meses, com queixas vocais variadas, provenientes de diversos consultórios, clínicas, ambulatórios e hospitais.

$\mathrm{O}$ QVV é um protocolo de rápido e fácil preenchimento, com dez questões em dois domínios: físico (itens 1,2,3,6,7,9) e sócio-emocional (itens $4,5,8,10$ ). O protocolo possibilita o cálculo de três escores: um total e dois relativos a cada domínio, variando de 0 a 100, com a pontuação máxima indicando melhor qualidade de vida relacionada à voz.

Para o cálculo dos escores é empregada a seguinte fórmula, um algoritmo padrão desse tipo de questionário:

Escore total: $100-\frac{(\text { escore bruto }-10)}{40} \times 100$

Escore físico: $100-\frac{(\text { escore físico - 6) }}{24}$ x100

Escore sócio-emocional: $100-\frac{(\text { escore sócio-emocional - 4) }}{16} \times 100$

Para a verificação do impacto de uma disfonia na qualidade de vida de acordo com idade, foram consideradas as seguintes faixas etárias: de 14 a 19 anos, 20 a 29 anos, 30 a 39 anos, 40 a 49 anos, 50 a 59 anos e 60 anos e mais.

O uso vocal profissional foi analisado com base na classificação sugerida em estudo anterior ${ }^{(23)}$, em que as profissões se dividem em quatro níveis de acordo com a demanda e importância da voz no trabalho: nível I - elite vocal (cantores e atores profissionais) - nesse caso até uma alteração de grau discreto pode prejudicar seriamente a carreira; nível II - usuário profissional da voz (professores, conferencistas, padres) - apenas uma alteração de grau moderado acarreta consequências mais sérias; nível III - usuário não-profissional vocal (comerciantes, médicos, advogados) - somente uma alteração de grau severo comprometeria a carreira; nível IV - usuário não-profissional não-vocal (programadores de computação, operários) - nem uma alteração de grau severo impede o trabalho.

Para a análise estatística foram utilizados o teste t de Student na comparação dos escores total, físico e sócio-emocional do QVV, para a identificação de possíveis diferenças entre ambos os gêneros. A Análise de Variância foi utilizada para a identificação de possíveis diferenças entre todas as faixas etárias e os níveis de uso vocal profissional. Foi ainda realizada uma comparação par a par nos domínios onde foi obtida significância entre as variáveis estudadas, também por meio de Análise de Variância. O nível de significância adotado foi de $5 \%(p \leq 0,05)$.

\section{RESULTADOS}

A população estudada foi de 1304 indivíduos, 996 do gênero feminino e 308 do gênero masculino, com idades entre 14 e 80 anos e média de 34 anos e cinco meses, sendo a maioria 
Tabela 1. Caracterização da população de acordo com gênero por faixa etária e por uso vocal profissional

\begin{tabular}{|c|c|c|c|c|c|c|}
\hline \multirow[t]{2}{*}{ Caracterização } & \multicolumn{2}{|c|}{ Feminino } & \multicolumn{2}{|c|}{ Masculino } & \multicolumn{2}{|c|}{ Total } \\
\hline & $\mathrm{N}$ & $\%$ & $\mathrm{~N}$ & $\%$ & $\mathrm{~N}$ & $\%$ \\
\hline \multicolumn{7}{|l|}{ Faixa etária } \\
\hline $14-19$ & 59 & 5,9 & 19 & 6,1 & 78 & 5,9 \\
\hline $20-29$ & 372 & 37,3 & 96 & 31,1 & 468 & 35,8 \\
\hline $30-39$ & 283 & 28,4 & 79 & 25,4 & 362 & 27,7 \\
\hline $40-49$ & 182 & 18,2 & 45 & 14,6 & 227 & 17,4 \\
\hline $50-59$ & 68 & 6,8 & 35 & 11,3 & 103 & 7,8 \\
\hline$\geq 60$ & 32 & 3,2 & 34 & 11,0 & 66 & 5,0 \\
\hline Total & 996 & 100 & 308 & 100 & 1304 & 100 \\
\hline \multicolumn{7}{|c|}{ Uso vocal profissional } \\
\hline Nível I & 36 & 3,6 & 31 & 10,0 & 67 & 5,1 \\
\hline Nível II & 406 & 40,7 & 78 & 25,3 & 484 & 37,1 \\
\hline Nível III & 275 & 27,6 & 111 & 36,0 & 386 & 29,6 \\
\hline Nível IV & 279 & 28,0 & 88 & 28,5 & 367 & 28,1 \\
\hline Total & 996 & 100 & 308 & 100 & 1304 & 100 \\
\hline
\end{tabular}

pertencente ao nível II de uso profissional da voz. Pode-se observar a caracterização da população estudada na Tabela 1.

$\mathrm{Na}$ Tabela 2 observa-se a comparação dos escores total, físico e sócio-emocional entre os gêneros. Os resultados não mostraram diferenças entre homens e mulheres (domínio total $\mathrm{p}=0,708$, domínio físico $\mathrm{p}=0,717$ e domínio sócio-emocional $\mathrm{p}=0,904)$, indicando que o impacto de uma alteração vocal na qualidade de vida é percebido de maneira semelhante por ambos os gêneros.

Tabela 2. Comparação dos escores médios obtidos no protocolo QVV por gênero nos três domínios

\begin{tabular}{lccc}
\hline Gênero & Domínio total & Domínio físico & $\begin{array}{c}\text { Domínio sócio- } \\
\text { emocional }\end{array}$ \\
\hline Feminino & 74,97 & 70,79 & 82,16 \\
Masculino & 75,50 & 71,37 & 82,34 \\
\hline Valor de $\mathrm{p}$ & 0,708 & 0,717 & 0,904 \\
\hline
\end{tabular}

Teste t de Student

Por meio da análise realizada entre as faixas etárias (Tabela 3), observa-se que existe diferença entre os três domínios (considerando os escores das diferentes faixas etárias). $\mathrm{Na}$ Tabela 4 vê-se a comparação par a par, por faixas etárias, dos escores relativos aos domínios total, físico e sócio-emocional, sendo que nos três domínios foram encontradas as mesmas diferenças. De acordo com as Tabelas 3 e 4 pode-se dizer que os indivíduos de 14 a 19 anos apresentaram escores menores que os de 20 a 29 anos (no domínio total p=0,006, no físico $\mathrm{p}=0,009$ e no sócio-emocional $\mathrm{p}=0,035$ ), os de 20 a 29 anos apresentaram escores mais elevados (total de 82,22, físico de 77,88 e sócio-emocional de 89,21) que os indivíduos de todas as outras faixas etárias e os de 30 a 39 anos apresentaram escores mais altos (total de 74,87, físico de 71,75 e sócio-emocional de 81,86$)$ que os de 40 a 49 anos nos domínios total e físico ( $\mathrm{p}=0,014$ e $\mathrm{p}=0,012$ respectivamente) e também que os de 60 anos e mais, nos três domínios (total $\mathrm{p}=0,025$, físico $\mathrm{p}=0,020$ e sócio-emocional p=0,041). Na comparação múltipla entre as faixas etárias de 40 a 49 anos, 50 a 59 anos e 60 anos e mais não foi encontrada diferença estatística $(\mathrm{p}>0,05)$.

Tabela 3. Comparação entre os escores médios obtidos no protocolo QVV por faixa etária nos três domínios

\begin{tabular}{lccc}
\hline Faixa etária & Domínio total & Domínio físico & $\begin{array}{c}\text { Domínio sócio- } \\
\text { emocional }\end{array}$ \\
\hline $14-19$ & 74,49 & 69,42 & 82,29 \\
$20-29$ & 82,22 & 77,88 & 89,21 \\
$30-39$ & 74,87 & 71,75 & 81,86 \\
$40-49$ & 68,16 & 63,11 & 76,14 \\
$50-59$ & 66,99 & 62,11 & 73,53 \\
$\geq 60$ & 62,79 & 59,34 & 68,54 \\
\hline Total & 75,09 & 70,93 & 82,20 \\
\hline Valor de p & $<0,001^{*}$ & $<0,001^{*}$ & $<0,001^{*}$ \\
\hline Análise de Variância & & & \\
* Valores significativos $(p \leq 0,05)$ & &
\end{tabular}

A Tabela 5 mostra a comparação dos escores total, físico e sócio-emocional entre os diversos níveis de uso vocal profissional e a Tabela 6 mostra a comparação par a par nos domínios total e físico, nos quais se obteve diferença significativa. O nível I, elite vocal, composto por atores e cantores, foi aquele no qual os indivíduos referiram menor impacto na qualidade de vida por uma disfonia nos domínios total e físico (escores médios no domínio total de 80,93 e no domínio físico de 80,97). Os profissionais dos níveis II, III e IV apresentaram escores estatisticamente semelhantes nos três domínios ( $p>0,005$ na comparação par a par entre estes grupos), indicando que, independente da grande demanda e risco vocal no uso de voz falada sem características artísticas para o trabalho, professores, operadores de teleatendimento e conferencistas percebem um problema de voz na qualidade de vida da mesma maneira que advogados ou programadores de computação, que possuem baixa ou nenhuma demanda ou 
Tabela 4. Comparação par a par, entre faixas etárias, dos escores médios nos domínios do protocolo QVV

\begin{tabular}{lccc}
\hline Faixas etárias & $\begin{array}{c}\text { Domínio total } \\
\text { (Valor de } p)\end{array}$ & $\begin{array}{c}\text { Domínio físico } \\
(\text { Valor de } p)\end{array}$ & $\begin{array}{c}\text { Domínio sócio- } \\
\text { emocional } \\
\text { (Valor de } p)\end{array}$ \\
\hline $14-19 \times 20-29$ & $0,006^{*}$ & $0,009^{*}$ & $0,035^{*}$ \\
$14-19 \times 30-39$ & 1,000 & 1,000 & 1,000 \\
$14-19 \times 40-49$ & 0,192 & 0,367 & 0,270 \\
$14-19 \times 50-59$ & 0,310 & 0,485 & 0,179 \\
$14-19 \times \geq 60$ & 0,067 & 0,178 & 0,056 \\
$20-29 \times 30-39$ & $<0,001^{*}$ & $<0,001^{*}$ & $<0,001^{*}$ \\
$20-29 \times 40-49$ & $<0,001^{*}$ & $<0,001^{*}$ & $<0,001^{*}$ \\
$20-29 \times 50-59$ & $<0,001^{*}$ & $<0,001^{*}$ & $<0,001^{*}$ \\
$20-29 \times \geq 60$ & $<0,001^{*}$ & $<0,001^{*}$ & $<0,001^{*}$ \\
$30-39 \times 40-49$ & $0,014^{*}$ & $0,012^{*}$ & 0,071 \\
$30-39 \times 50-59$ & 0,108 & 0,063 & 0,103 \\
$30-39 \times \geq 60$ & $0,025^{*}$ & $0,020^{*}$ & $0,041^{*}$ \\
$40-49 \times 50-59$ & 1,000 & 1,000 & 1,000 \\
$40-49 \times \geq 60$ & 0,932 & 0,997 & 0,746 \\
$50-59 \times \geq 60$ & 0,998 & 1,000 & 0,996 \\
\hline Ana & & &
\end{tabular}

Análise de Variância

* Valores significativos $(p \leq 0,05)$

risco vocal em suas profissões. Os escores sócio-emocionais de todos os grupos foram estatisticamente similares, não revelando aspectos particulares da população estudada.

Tabela 5. Comparação entre os escores médios obtidos no protocolo QVV por nível de uso vocal nos três domínios

\begin{tabular}{lccc}
\hline $\begin{array}{l}\text { Uso vocal } \\
\text { profissional }\end{array}$ & Domínio total & Domínio físico & $\begin{array}{c}\text { Domínio sócio- } \\
\text { emocional }\end{array}$ \\
\hline Nível I & 80,93 & 80,97 & 81,81 \\
Nível II & 73,25 & 69,09 & 81,85 \\
Nível III & 76,51 & 71,48 & 83,62 \\
Nível IV & 75,01 & 71,01 & 81,28 \\
\hline Total & 75,11 & 70,95 & 82,21 \\
\hline Valor de p & $0,025^{*}$ & $0,010^{*}$ & 0,0537 \\
\hline
\end{tabular}

Análise de Variância

* Valores significativos $(p \leq 0,05)$

Tabela 6. Comparação par a par, entre os níveis de uso vocal profissional, dos escores médios nos domínios do protocolo QVV

\begin{tabular}{lcc}
\hline Níveis & $\begin{array}{c}\text { Domínio total } \\
(\text { Valor de } p)\end{array}$ & $\begin{array}{c}\text { Domínio físico } \\
\text { (Valor de } p)\end{array}$ \\
\hline I x II & $0,006^{*}$ & $<0,001^{*}$ \\
I x III & 0,312 & $<0,001^{*}$ \\
I x IV & 0,072 & $<0,001^{*}$ \\
II x III & 0,205 & 0,783 \\
II x IV & 0,834 & 0,892 \\
III x IV & 0,934 & 1,000 \\
\hline
\end{tabular}

Análise de Variância

${ }^{*}$ Valores significativos $(p \leq 0,05)$

\section{DISCUSSÃO}

Para a avaliação do impacto de uma alteração vocal em um indivíduo, os achados laringoscópicos, a descrição dos desvios vocais e a caracterização acústica não são suficientes, pois faltam informações importantes sobre como essa alteração interfere nas atividades diárias em contexto pessoal, social e profissional ${ }^{(26)}$. Os efeitos de uma alteração vocal na qualidade de vida têm sido um assunto bastante estudado nas últimas décadas ${ }^{(3,5,5,10)}$, porém há poucas informações indicando se há diferença na forma como este impacto é percebido de acordo com o gênero, idade e uso vocal profissional.

A partir dos resultados da comparação entre os gêneros (Tabela 2), pode-se dizer que o impacto de uma disfonia na qualidade de vida é percebido de maneira semelhante por homens e mulheres, que apresentaram respectivamente escores médios no domínio total de 75,50 e 74,97 ( $\mathrm{p}=0,708$ ), no domínio físico de 71,37 e 70,79 ( $\mathrm{p}=0,717)$ e no domínio sócio-emocional de 82,34 e 82,16 ( $\mathrm{p}=0,904)$. O mesmo resultado também foi observado em outros estudos ${ }^{(5,6,10,11,13)}$. É interessante comentar que, embora mais mulheres busquem por ajuda fonoaudiológica em relação aos homens na clínica de voz, dado também encontrado neste estudo (996 mulheres e 308 homens), isso parece não estar relacionado ao impacto que a alteração vocal causa na qualidade de vida, já que na população estudada não houve diferença entre os grupos de acordo com o gênero. Contudo, deve-se ressaltar que o tipo e o grau da disfonia, fator que pode interferir nestes resultados, não foi levado em consideração nas análises.

Em relação à idade, pode-se observar em trabalhos anteriores $^{(11,13)}$, nos quais o QVV foi aplicado em populações com alterações vocais específicas, como indivíduos laringectomizados e com paralisia de prega vocal unilateral em tratamento cirúrgico de tireoplastia tipo I (antes da cirurgia), os mais jovens perceberam maior impacto da disfonia na qualidade de vida. Porém no pós-operatório dos indivíduos com paralisia unilateral de prega vocal, esta situação se inverteu e as pessoas acima de 65 anos apresentaram escores menores do que os mais jovens. Resultados semelhantes foram encontrados em outro trabalho que pesquisou uma grande população com e sem queixa vocal ${ }^{(5)}$. De acordo com o que foi encontrado no presente estudo, parece que a idade influencia a percepção da qualidade de vida relacionada à voz, mas não houve uma distribuição linear dos escores entre as faixas etárias extremas. Nas Tabelas 3 e 4 observa-se maiores escores nos três domínios na faixa etária de 20 a 29 anos (total de 82,22, físico de 77,88 e sócio-emocional de 89,21 ) em relação à todas as outras idades estudadas, indicando que para estes indivíduos um problema de voz não compromete a qualidade de vida, pois uma disfonia não traz grandes limitações para as atividades diárias e funções sociais. Este dado pode ser explicado, em parte, por esta ser uma população que está em uma fase ativa de sua vida com preocupações dirigidas principalmente para a profissão/carreira e inserção no mercado de trabalho e possivelmente um problema de voz passar a ter menor importância. Quando os indivíduos de 30 a 39 anos são comparados às populações com idade entre 40 e 49 anos e 60 anos e mais, observa-se que eles apresentam menor impacto na qualidade 
de vida. Os menores escores da população idosa (60 anos e mais) em relação à população de 20 a 29 anos e 30 a 39 anos podem ser parcialmente justificados pelo processo natural e global de envelhecimento, que produz mudanças anatômicas e fisiológicas em todo o organismo. A literatura indica que mulheres idosas (entre 60 e 103 anos) com uma alteração vocal de grau leve a moderado não percebem um impacto negativo na qualidade de vida relacionada à voz ${ }^{(20)}$. Em outra pesquisa viu-se que através da terapia vocal é possível reduzir o impacto do problema de voz na qualidade de vida da população com idade mais avançada ${ }^{(27)}$. É importante ressaltar a necessidade de trabalhos que investiguem a influência da idade na percepção da qualidade de vida relacionada à voz, considerando o tipo e grau de disfonia, uma vez que esta pesquisa considerou apenas a presença de queixa vocal.

A comunicação adquire um papel cada vez mais importante para os indivíduos que usam a voz profissionalmente ou buscam inserção no mercado de trabalho ${ }^{(25)}$. Alguns destes profissionais têm maior demanda e risco vocal, como os pertencentes aos níveis I e $\mathrm{II}^{(18,23)}$, sendo que os profissionais do nível I utilizam a voz artística (cantores e atores) e os profissionais do nível II a voz falada (professores, operadores de teleatendimento, conferencistas, padres), embora geralmente em maior quantidade de uso. Ambos os níveis de uso vocal profissional apresentam grande demanda e risco vocal em seus trabalhos, por isso espera-se que uma disfonia possa ser um agente limitante do bom rendimento profissional. Esta limitação pode implicar em faltas, queda de rendimento profissional, frustração e até mesmo no desejo de mudança de profissão._Contudo, os resultados foram surpreendentes. Atores e cantores, tiveram os maiores escores físicos ( $\mathrm{p}<0,001$ na comparação par a par com os outros três grupos - Tabela 6), que podiam ter sido obtidos, por que este protocolo não é específico para avaliar o impacto de uma disfonia no uso da voz profissional cantada e falada artística e, portanto, pode não ser sensível o suficiente para se detectar desvios. Outra hipótese é que talvez estes indivíduos considerem importante qualquer sintoma da disfonia, e por isso, recorram à ajuda especializada precocemente, de forma que o problema não chega a prejudicar a carreira ou a vida social $^{(5,28)}$. Já os profissionais do nível II tiveram escores (total de 73,25, físico de 69,09 e sócio-emocional de 81,85) estatisticamente semelhantes aos dos níveis III (76,51 total, 71,48 físico e 83,62 sócio-emocional) e IV (75,01 total, 71,01 físico e 81,28 sócio-emocional), que possuem menor demanda e risco de uso vocal (Tabelas 5 e 6 ). Muito provavelmente estes profissionais tenham dificuldade em relacionar os problemas de voz com o seu trabalho e em perceber o impacto deles na qualidade de vida, como visto em um estudo realizado com professores $^{(29)}$.

Por fim, é interessante ressaltar que, na comparação entre os domínios do protocolo, independente do uso de voz que um indivíduo tenha em sua profissão, os maiores impactos ocorrem no domínio físico (Tabelas 5 e 6), que indica a percepção do quanto a voz não está funcionando como deveria, seja por problemas na mecânica de sua produção, seja por problemas no uso diário da $\mathrm{voz}^{(5,25,30)}$.

Este estudo analisou 1304 protocolos de indivíduos brasileiros, com queixa e diagnóstico de alteração vocal, de ambos os gêneros, ampla faixa etária (de 14 a 80 anos), de diferentes profissões, categorizados em quatro níveis de uso de voz. Assim, os resultados encontrados podem auxiliar o fonoaudiólogo a comparar os dados de um paciente com problemas de voz em relação a uma grande amostra estudada, com informações sobre os valores esperados de acordo com a idade e o nível de uso vocal profissional. Um acréscimo importante seria analisar a influência do grau de alteração vocal avaliado clinicamente pelo fonoaudiólogo e percebido pelo sujeito com os resultados do protocolo de qualidade de vida em relação aos aspectos vocais.

\section{CONCLUSÃO}

O impacto auto-relatado na qualidade de vida relacionado a uma alteração vocal é percebido de maneira semelhante por homens e mulheres. Os indivíduos de 20 a 29 anos percebem o impacto da disfonia na sua qualidade de vida diferente dos indivíduos das outras faixas etárias. Os profissionais com grande demanda de voz falada artística relataram sofrer o menor impacto de um problema de voz na qualidade de vida.

\begin{abstract}
Purpose: To verify the self-reported impact of a vocal disorder on the quality of life of individuals with vocal complaints, according to gender, age and occupational use of voice. Methods: We analyzed 1034 Voice-Related Quality of Life (VRQOL) protocols answered by Brazilian individuals with varied vocal complaints. The subjects were 996 women and 308 men. Statistical analyses compared the variables gender, age and level of occupational use of voice. Results: Total results were similar for men (total 75.5; physical 71.3; socio-emotional 82.3) and women (total 74.9; physical 70.7; socio-emotional 82.1) in the three domains. Higher VRQOL scores were observed for individuals with ages between 20 and 29 years (total 82.2; physical 77.8; socio-emotional 89.2). Conclusion: The self-reported impact of a vocal disorder in the quality of life was similarly noticed by men and women. The subjects aged between 20 and 29 years had different perceptions regarding the impact of dysphonia in their quality of life when compared to individuals of the other age ranges. Vocal disorders caused less impact on the quality of life of elite vocal performers.
\end{abstract}

Keywords: Voice; Quality of life; Voice disorders; Dysphonia; Sickness impact profile; Health care; Health evaluation; Age effect 


\section{REFERÊNCIAS}

1. World Health Organization. Programme on Mental Health. WHOQOL. Measuring Quality of Life. Geneva: World Health Organization; 1997. p.1-5.

2. Berlim MT, Fleck MPA. Quality of life: a brand new concept for research and practice in psychiatry. Rev Bras Psiquiatr. 2003;25(4):24952.

3. Hogikyan ND, Rosen CA. A review of outcome measurements for voice disorders. Otolaryngol Head Neck Surg. 2002;126(5):562-72. Review.

4. Higginson IJ, Carr AJ. Measuring quality of life: Using quality of life measures in the clinical setting. BMJ. 2001;322(7297):1297-300.

5. Behlau M, Hogikyan ND, Gasparini G. Quality of life and voice: study of a Brazilian population using the voice-related quality of life measure. Folia Phoniatr Logop. 2007;59(6):286-96.

6. Krischke S, Weigelt S, Hoppe U, Köllner V, Klotz M, Eysholdt U, Rosanowski F. Quality of life in dysphonic patients. J Voice. 2005;19(1):132-7.

7. Portone CR, Hapner ER, McGregor L, Otto K, Johns MM 3rd. Correlation of the Voice Handcap Index (VHI) and the Voice-Related Quality of Life Measure (V-RQOL). J Voice. 2007;21(6):723-7.

8. Hogikyan ND, Sethuraman G. Validation of an instrument to measure voice-related quality of life (V-RQOL). J Voice. 1999;13(4):557-69.

9. Kasama ST, Brasolotto AG. Percepção vocal e qualidade de vida. PróFono. 2007;19(1):19-28.

10. Gasparini G, Behlau M. Quality of life: validation of the Brazilian version of the voice-related quality of life (V-RQOL) measure. J Voice. 2009;23(1):76-81.

11. Hogikyan ND, Wodchis WP, Terrell JE, Bradford CR, Esclamado RM. Voice-related quality of life (V-RQOL) following type I thyroplasty for unilateral vocal folds paralysis. J Voice. 2000;14(3):378-86.

12. Behlau M, Madázio G, Feijó D, Pontes P. Avaliação de voz. In: Behlau M. Voz: o livro do especialista. Rio de Janeiro: Revinter; c2001. Vol. 1. Cap.3. p. 85-245.

13. Kazi R, De Cordova J, Singh A, Venkitaraman R, Nutting CM, Clarke P, et al. Voice-related Quality of Life in laringectomees: assessment using the VHI and V-RQOL symptom scales. J Voice. 2007;21(6):728-34.

14. Hogikyan ND, Wodchis WP, Spak C, Kileny PR. Longitudinal effects of botulinum toxin injections on voice-related quality of life (V-RQOL) for patients with adductory spasmodic dysphonia. J Voice. 2001;15(4):57686.

15. Rubin AD, Wodchis WP, Spak C, Kileny PR, Hogikyan ND. Longitudinal effects of Botox injections on voice-related quality of life (V-RQOL) for patients with adductory spasmodic dysphonia: part II. Arch Otolaryngol Head Neck Surg. 2004;130(4):415-20.
16. Boseley ME, Cunningham MJ, Volk MS, Hartnick CJ. Validation of the Pediatric Voice-Related Quality-of-Life survey. Arch Otolaryngol Head Neck Surg. 2006;132(7):717-20.

17. Hartnick CJ, Volk MS, Cunningham M. Establishing normative voice-related quality of life scores within the pediatric otolarygology population. Arch Otolaryngol Head Neck Surg. 2003;129(10):1090-3.

18. Behlau M, Feijó D, Madázio G, Rehder MI, Azevedo R, Ferreira AE. Voz profissional: aspectos gerais e atuação fonoaudiológica. In: Behlau M. Voz: o livro do especialista. Rio de Janeiro: Revinter; 2005. V. 2. cap. 12. p. 287-407.

19. Behlau M, Azevedo R, Pontes P. Conceito de voz normal e classificação das disfonias. In: Behlau M. Voz: o livro do especialista. Rio de Janeiro: Revinter; 2004. cap. 2. p. 53-84.

20. Gama ACC, Alves CFT, Cerceau JSB, Teixeira LC. Correlação entre dados perceptivo-auditivos e qualidade de vida em voz de idosas. PróFono. 2009;21(2):125-30.

21. Vilkman E. Occupational safety and health aspects of voice and speech professions. Folia Phoniatr Logop. 2004;56(4):220-53.

22. Roy N, Gray SD, Simon M, Dove H, Corbin-Lewis K, Stemple JC. An evaluation of the effects of two treatment approaches for teachers with voice disorders: a prospective randomized clinical trial. J Speech Lang Hear Res. 2001;44(2):286-96.

23. Koufman JA, Isaacson G. Voice disorders. Otolaryngol Clin North Am. 1991;24(5):989-98.

24. Jones K, Sigmon J, Hock L, Nelson E, Sullivan M, Ogren F. Prevalence and risk factors for voice problems among telemarketers. Arch Otolaryngol Head Neck Surg. 2002;128(5):571-7.

25. Fortes FSG, Imamura R, Tsuji DH, Sennes LU. Perfis dos profissionais da voz com queixas vocais atendidos em um centro terciário de saúde. Rev Bras Otorrinolaringol. 2007;73(1):27-31.

26. Ma EP, Yiu EM. Voice activity and participation profile: assessing the impact of voice disorders on daily activities. J Speech Lang Hear Res. 2001;44(3):511-24.

27. Berg EE, Harpner E, Klein A, Johns MM 3rd. Voice therapy improves quality of life in age-related dysphonia: a case-control study. J Voice. 2008;22(1):70-4.

28. Rosen CA, Murry T. Voice handicap index in singers. J Voice. 2000;14(3):370-7.

29. Grillo MHMM, Penteado RZ. Impacto da voz na qualidade de vida de professore(a)s do ensino fundamental. Pró-Fono. 2005;17(3):321-30.

30. Behlau M, Oliveira G. Self-assessment of dysphonia in professional and non professional voice users - the Brazilian experience. XIX Ent World Congress IFOS 2009 Brasil. 